\title{
Uniform Asymptotic Expansion for the Incomplete Beta Function
}

\author{
Gergő NEMES and Adri B. OLDE DAALHUIS
}

Maxwell Institute and School of Mathematics, The University of Edinburgh, Peter Guthrie Tait Road, Edinburgh EH9 3FD, UK

E-mail:Gergo.Nemes@ed.ac.uk,A.B.Olde.Daalhuis@ed.ac.uk

URL: http://www.maths.ed.ac.uk/ gnemes/, http://www.maths.ed.ac.uk/ adri/

Received September 12, 2016, in final form October 21, 2016; Published online October 25, 2016

http://dx.doi.org/10.3842/SIGMA.2016.101

\begin{abstract}
In [Temme N.M., Special functions. An introduction to the classical functions of mathematical physics, A Wiley-Interscience Publication, John Wiley \& Sons, Inc., New York, 1996, Section 11.3.3.1] a uniform asymptotic expansion for the incomplete beta function was derived. It was not obvious from those results that the expansion is actually an asymptotic expansion. We derive a remainder estimate that clearly shows that the result indeed has an asymptotic property, and we also give a recurrence relation for the coefficients.
\end{abstract}

Key words: incomplete beta function; uniform asymptotic expansion

2010 Mathematics Subject Classification: 41A60; 33B20

\section{Introduction}

For positive real numbers $a, b$ and $x \in[0,1]$, the (normalised) incomplete beta function $I_{x}(a, b)$ is defined by

$$
I_{x}(a, b)=\frac{1}{B(a, b)} \int_{0}^{x} t^{a-1}(1-t)^{b-1} \mathrm{~d} t
$$

where $B(a, b)$ denotes the ordinary beta function:

$$
B(a, b)=\int_{0}^{1} t^{a-1}(1-t)^{b-1} \mathrm{~d} t=\frac{\Gamma(a) \Gamma(b)}{\Gamma(a+b)}
$$

(see, e.g., [2, Section 8.17(i)]). In this paper, we will use the notation of [2, Section 8.18(ii)].

The incomplete beta function plays an important role in statistics in connection with the beta distribution (see, for instance, [1, pp. 210-275]). Large parameter asymptotic approximations are useful in these applications. For fixed $x$ and $b$, one could use the asymptotic expansion

$$
I_{x}(a, b)=\frac{x^{a}(1-x)^{b-1}}{a B(a, b)}{ }_{2} F_{1}\left(\begin{array}{c}
1,1-b \\
a+1
\end{array} ; \frac{x}{x-1}\right) \sim \frac{x^{a}(1-x)^{b-1}}{a B(a, b)} \sum_{n=0}^{\infty} \frac{(1-b)_{n}}{(a+1)_{n}}\left(\frac{x}{x-1}\right)^{n} \text {, }
$$

as $a \rightarrow+\infty$. The right-hand side of (1) converges only for $x \in\left[0, \frac{1}{2}\right)$, but for any fixed $x \in[0,1)$ it is still useful when used as an asymptotic expansion as $a \rightarrow+\infty$. For more details, see [3, Section 11.3.3]. However, it is readily seen that (1) breaks down as $x \rightarrow 1$. Since this limit has significant importance in applications, Temme derived in [3, Section 11.3.3.1] an asymptotic expansion as $a \rightarrow+\infty$ that holds uniformly for $x \in(0,1]$. His result can be stated as follows. 
Theorem 1. Let $\xi=-\ln x$. Then for any fixed positive integer $N$ and fixed positive real $b$,

$$
I_{x}(a, b)=\frac{\Gamma(a+b)}{\Gamma(a)}\left(\sum_{n=0}^{N-1} d_{n} F_{n}+\mathcal{O}\left(a^{-N}\right) F_{0}\right),
$$

as $a \rightarrow+\infty$, uniformly for $x \in(0,1]$. The functions $F_{n}=F_{n}(\xi, a, b)$ are defined by the recurrence relation

$$
a F_{n+1}=(n+b-a \xi) F_{n}+n \xi F_{n-1},
$$

with

$$
F_{0}=a^{-b} Q(b, a \xi), \quad F_{1}=\frac{b-a \xi}{a} F_{0}+\frac{\xi^{b} \mathrm{e}^{-a \xi}}{a \Gamma(b)}
$$

and $Q(a, z)=\Gamma(a, z) / \Gamma(a)$ is the normalised incomplete gamma function (see [2, Section 8.2(i)]). The coefficients $d_{n}=d_{n}(\xi, b)$ are defined by the generating function

$$
\left(\frac{1-\mathrm{e}^{-t}}{t}\right)^{b-1}=\sum_{n=0}^{\infty} d_{n}(t-\xi)^{n}
$$

In particular,

$$
d_{0}=\left(\frac{1-x}{\xi}\right)^{b-1}, \quad d_{1}=\frac{x \xi+x-1}{(1-x) \xi}(b-1) d_{0} .
$$

They satisfy the recurrence relation

$$
\begin{aligned}
\xi(n+1)(n+2) d_{0} d_{n+2}= & \xi \sum_{m=0}^{n}(m+1)\left(n-2 m+1+\frac{m-n-1}{b-1}\right) d_{m+1} d_{n-m+1} \\
& +\sum_{m=0}^{n}(m+1)\left(n-2 m-2-\xi+\frac{m-n}{b-1}\right) d_{m+1} d_{n-m} \\
& +\sum_{m=0}^{n}(1-m-b) d_{m} d_{n-m} .
\end{aligned}
$$

In the case that $b=1$, we have $d_{0}=1$ and $d_{n}=0$ for $n \geq 1$.

Our contribution is the remainder estimate in (2) and the recurrence relation (5). In fact, it is not at all obvious from (3) that the sequence $\left\{F_{n}\right\}_{n=0}^{\infty}$ has an asymptotic property as $a \rightarrow+\infty$. We will show that for any non-negative integer $n$,

$$
0<F_{n+1} \leq \frac{n+\beta}{a} F_{n}
$$

where $\beta=\max (1, b)$.

In [4, Section 38.2.8] the function $F_{n}$ is identified as a Kummer $U$-function:

$$
F_{n}=\frac{\xi^{n+b} \mathrm{e}^{-a \xi} n !}{\Gamma(b)} U(n+1, n+b+1, a \xi)
$$




\section{Proof of the main results}

We proceed similarly as in [3, Section 11.3 .3 .1$]$ and start with the integral representation

$$
I_{x}(a, b)=\frac{1}{B(a, b)} \int_{\xi}^{+\infty} t^{b-1} \mathrm{e}^{-a t}\left(\frac{1-\mathrm{e}^{-t}}{t}\right)^{b-1} \mathrm{~d} t .
$$

We substitute the truncated Taylor series expansion

$$
\left(\frac{1-\mathrm{e}^{-t}}{t}\right)^{b-1}=\sum_{n=0}^{N-1} d_{n}(t-\xi)^{n}+r_{N}(t)
$$

into (7) and obtain

$$
I_{x}(a, b)=\frac{\Gamma(a+b)}{\Gamma(a)}\left(\sum_{n=0}^{N-1} d_{n} F_{n}+R_{N}(a, b, x)\right),
$$

where $F_{n}$ is given by the integral representation

$$
F_{n}=\frac{1}{\Gamma(b)} \int_{\xi}^{+\infty} t^{b-1} \mathrm{e}^{-a t}(t-\xi)^{n} \mathrm{~d} t=\frac{\mathrm{e}^{-a \xi}}{\Gamma(b)} \int_{0}^{+\infty}(\tau+\xi)^{b-1} \tau^{n} \mathrm{e}^{-a \tau} \mathrm{d} \tau
$$

and the remainder term $R_{N}(a, b, x)$ is defined by

$$
R_{N}(a, b, x)=\frac{1}{\Gamma(b)} \int_{\xi}^{+\infty} t^{b-1} \mathrm{e}^{-a t} r_{N}(t) \mathrm{d} t .
$$

The recurrence relation (3) can be obtained from (8) via a simple integration by parts.

Let, for a moment,

$$
c_{n}(a, b)=\int_{0}^{+\infty}(\tau+\xi)^{b-1} \tau^{n} \mathrm{e}^{-a \tau} \mathrm{d} \tau .
$$

Then via integration by parts we find

$$
a c_{n+1}(a, b)=(n+b) c_{n}(a, b)+\xi(1-b) c_{n}(a, b-1)
$$

We make the observation that

$$
0 \leq \xi c_{n}(a, b-1)=\xi \int_{0}^{+\infty}(\tau+\xi)^{b-2} \tau^{n} \mathrm{e}^{-a \tau} \mathrm{d} \tau \leq c_{n}(a, b) .
$$

It follows from (10) and (11) that

$$
a c_{n+1}(a, b) \leq \begin{cases}(n+1) c_{n}(a, b) & \text { if } 0<b \leq 1, \\ (n+b) c_{n}(a, b) & \text { if } b \geq 1 .\end{cases}
$$

Since $F_{n}=\mathrm{e}^{-a \xi} c_{n}(a, b) / \Gamma(b)$, this inequality implies (6).

To obtain the remainder estimate in (2), we use the Cauchy integral representation

$$
r_{N}(t)=\frac{(t-\xi)^{N}}{2 \pi \mathrm{i}} \oint_{\{\xi, t\}} \frac{\left(\frac{1-\mathrm{e}^{-\tau}}{\tau}\right)^{b-1}}{(\tau-t)(\tau-\xi)^{N}} \mathrm{~d} \tau,
$$


where the contour encircles the points $\xi$ and $t$ once in the positive sense. From the integral representation (9), we have that $0 \leq \xi \leq t$. Thus, in the case that $N \geq 1$, we can deform the contour in (12) to the path

$$
\begin{aligned}
{[1+\infty \mathrm{i}, 1+\pi \mathrm{i}] } & \cup[1+\pi \mathrm{i},-1+\pi \mathrm{i}] \cup[-1+\pi \mathrm{i},-1-\pi \mathrm{i}] \\
& \cup[-1-\pi \mathrm{i}, 1-\pi \mathrm{i}] \cup[1-\pi \mathrm{i}, 1-\infty \mathrm{i}] .
\end{aligned}
$$

For the integrals along the final three portions of the path, we have the estimates

$$
\begin{aligned}
& \left|\frac{1}{2 \pi \mathrm{i}} \int_{-1+\pi \mathrm{i}}^{-1-\pi \mathrm{i}} \frac{\left(\frac{1-\mathrm{e}^{-\tau}}{\tau}\right)^{b-1}}{(\tau-t)(\tau-\xi)^{N}} \mathrm{~d} \tau\right| \leq \frac{\max \left((\mathrm{e}-1)^{b-1},\left(\frac{\mathrm{e}+1}{\sqrt{\pi^{2}+1}}\right)^{b-1}\right)}{(1+\xi)^{N+1}}, \\
& \left|\frac{1}{2 \pi \mathrm{i}} \int_{-1-\pi \mathrm{i}}^{1-\pi \mathrm{i}} \frac{\left(\frac{1-\mathrm{e}^{-\tau}}{\tau}\right)^{b-1}}{(\tau-t)(\tau-\xi)^{N}} \mathrm{~d} \tau\right| \leq \frac{\max \left(\left(\frac{\mathrm{e}^{ \pm 1}+1}{\sqrt{\pi^{2}+1}}\right)^{b-1}\right)}{\pi^{N+2}},
\end{aligned}
$$

and

$$
\begin{aligned}
\left|\frac{1}{2 \pi \mathrm{i}} \int_{1-\pi \mathrm{i}}^{1-\infty \mathrm{i}} \frac{\left(\frac{1-\mathrm{e}^{-\tau}}{\tau}\right)^{b-1}}{(\tau-t)(\tau-\xi)^{N}} \mathrm{~d} \tau\right| & \leq \frac{1}{2 \pi} \int_{\pi}^{+\infty} \frac{\max \left(\left(1 \pm \mathrm{e}^{-1}\right)^{b-1}\right)\left(s^{2}+1\right)^{(1-b) / 2}}{\sqrt{s^{2}+(1-t)^{2}}\left(s^{2}+(1-\xi)^{2}\right)^{N / 2}} \mathrm{~d} s \\
& \leq \frac{\max \left(\left(1 \pm \mathrm{e}^{-1}\right)^{b-1}\right)}{2 \pi} \int_{\pi}^{+\infty} \frac{\left(s^{2}+1\right)^{(1-b) / 2}}{s^{N+1}} \mathrm{~d} s
\end{aligned}
$$

respectively. The integrals along the first two portions can be estimated similarly to (13) and (14). Hence, for $0 \leq \xi \leq t$ and $N \geq 1$, we have

$$
\left|r_{N}(t)\right| \leq C_{N}(b)(t-\xi)^{N},
$$

where the constant $C_{N}(b)$ does not depend on $\xi$. Using this result in the integral representation (9), we can infer that

$$
\left|R_{N}(a, b, x)\right| \leq C_{N}(b) F_{N} .
$$

Finally, combining this result with the inequalities (6), we obtain the required remainder estimate in $(2)$.

The reader can check that the function $f(t)=\left(\frac{1-\mathrm{e}^{-t}}{t}\right)^{b-1}$ is a solution of the nonlinear differential equation

$$
t f(t) f^{\prime \prime}(t)-\frac{b-2}{b-1} t f^{\prime 2}(t)+(t+2) f(t) f^{\prime}(t)+(b-1) f^{2}(t)=0 .
$$

If we substitute the Taylor series (4) into this differential equation and rearrange the result, we obtain the recurrence relation (5).

\section{Acknowledgements}

This research was supported by a research grant (GRANT11863412/70NANB15H221) from the National Institute of Standards and Technology. The authors thank the anonymous referees for their helpful comments and suggestions on the manuscript. 


\section{References}

[1] Johnson N.L., Kotz S., Balakrishnan N., Continuous univariate distributions, Vol. 2, 2nd ed., Wiley Series in Probability and Mathematical Statistics: Applied Probability and Statistics, John Wiley \& Sons, Inc., New York, 1995.

[2] Olver F.W.J., Lozier D.W., Boisvert R.F., Clark C.W. (Editors), NIST handbook of mathematical functions, U.S. Department of Commerce, National Institute of Standards and Technology, Washington, DC, Cambridge University Press, Cambridge, 2010, Release 1.0.13 of 2016-09-16, available at http://dlmf.nist. gov/.

[3] Temme N.M., Special functions. An introduction to the classical functions of mathematical physics, $A$ WileyInterscience Publication, John Wiley \& Sons, Inc., New York, 1996.

[4] Temme N.M., Asymptotic methods for integrals, Series in Analysis, Vol. 6, World Scientific Publishing Co. Pte. Ltd., Hackensack, NJ, 2015. 